\title{
CCD photometry of XX Cyg
}

\author{
S. N. Udovichenko \\ Astronomical Observatory, Odessa National University, \\ Shevchenko Park, 65014, Odessa, Ukraine
}

XX Cyg ( $\alpha=20 \mathrm{~h} \mathrm{02m} \mathrm{17s,} \delta=58$ 48' 44" (1950), V=12 mag, A5) is well known as metal-poor, high-velocity SX Phe star (McNamara \& Feltz, 1980). It shows a period of light variation of $0.13486507 \mathrm{~d}$, and an amplitude up to $1 \mathrm{mag}$ (from the General Catalog of Variable Stars). The photometric observation of XX Cyg in different years show the variation of amplitude and period during a long time (Zhou et al., 2002). New observations of XX Cyg were made in 7

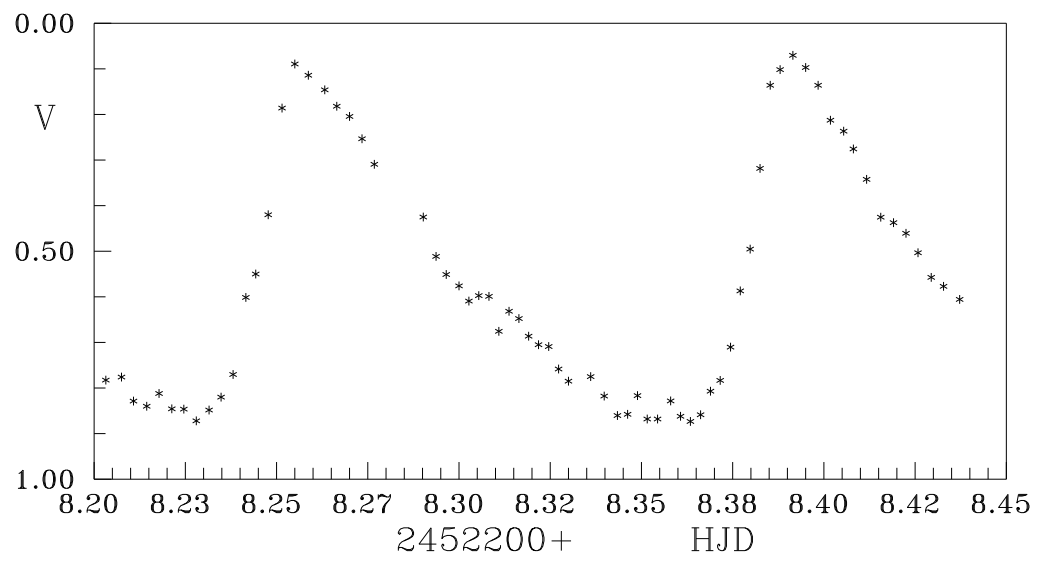

Figure 1: The light curve of XX Cyg (V) 25-26 October 2001.

nights of October 2001 and were continued in 2002 using the $48 \mathrm{~cm}$ reflector at the Astronomical Observatory of Odessa National University. One star $(\alpha=20 \mathrm{~h}$ $\left.02 \mathrm{~m} \mathrm{24s,} \delta=5851^{\prime} 43^{\prime \prime}\right)$ was used as a comparison star and monitored in the frame simultaneously. The CCD photometer was created using a chip ISD015 (520x580 pixels), vacuum housing and thermoelectric (Peltier) cooler. In the observations the $\mathrm{V}$ filter of the UBV system was used. 
A typical observed light curve of $X X$ Cyg is shown in Figure 1. A preliminary analysis of the light curve shows a total light range of $0.8 \mathrm{mag}$ in the filter $\mathrm{V}$. Possibly, the light curve shows, besides the primary maximum, a small bump of an amplitude of approximately $0.1 \mathrm{mag}$. The light variation of XX Cyg can be fit with a single pulsation frequency, but an analysis on multiple mode pulsation is being carried out.

\section{References}

Zhou, Ai-Ying, Jiang, Shi-Yang, Chayan, B., Du, Bai-Tian 2002, Ap\&SS 281(4) McNamara, D. H., Feltz, K. A., Jr. 1980, PASP 92 